\title{
Pier Massimo Prosio, Dalla Ginevra di Rousseau alla Londra di Dickens. Luogo e opera nella letteratura romantica
}

Marco Stupazzoni

\section{(2) OpenEdition \\ Journals}

Edizione digitale

URL: http://journals.openedition.org/studifrancesi/1523

DOI: $10.4000 /$ studifrancesi. 1523

ISSN: 2421-5856

\section{Editore}

Rosenberg \& Sellier

\section{Edizione cartacea}

Data di pubblicazione: 1 novembre 2014

Paginazione: 605-606

ISSN: 0039-2944

\section{Notizia bibliografica digitale}

Marco Stupazzoni, «Pier Massimo Prosio, Dalla Ginevra di Rousseau alla Londra di Dickens. Luogo e opera nella letteratura romantica », Studi Francesi [Online], 174 (LVIII | III) | 2014, online dal 01 novembre 2014, consultato il 18 septembre 2020. URL : http://journals.openedition.org/studifrancesi/1523 ; DOI : https://doi.org/10.4000/studifrancesi. 1523

Questo documento è stato generato automaticamente il 18 settembre 2020.

\section{cc) (†) $\odot$}

Studi Francesi è distribuita con Licenza Creative Commons Attribuzione - Non commerciale - Non opere derivate 4.0 Internazionale. 


\title{
Pier Massimo Prosio, Dalla Ginevra di Rousseau alla Londra di Dickens. Luogo e opera nella letteratura romantica
}

\author{
Marco Stupazzoni
}

\section{NOTIZIA}

PIER MASSIMO PROSIO, Dalla Ginevra di Rousseau alla Londra di Dickens. Luogo e opera nella letteratura romantica, Moncalieri, C.I.R.V.I., 2012, «Civilisation de l'Europe», pp. 234.

1 Nel vasto e suggestivo ambito degli studî di geografia letteraria collocabili nel panorama altrettanto esteso degli orizzonti nazionali ed internazionali, questo volume di Pier Massimo Prosio rappresenta uno dei possibili e originali esempî di una ricerca volta a mettere in luce il legame profondo tra un'opera letteraria ed un luogo geografico. In particolare, l'intenzione dell'autore è quella di svelare i significati che una determinata realtà geografica ha avuto in un determinato scritto e in quale misura essa abbia influito su quella determinata opera. Circoscrivendo i limiti del proprio lavoro alle opere di autori (a lui particolarmente cari) dell'epoca romantica (comprendendo anche Rousseau) e dell'Ottocento francese e inglese (con la sola eccezione italiana rappresentata dal piemontese Calandra), Prosio riesce brillantemente a dimostrare come, nel XIX secolo, tanto in letteratura quanto in pittura, il «rapporto con la terra e l'ambiente, l'attenzione per le particolarità di una specifica realtà regionale» siano «elementi rilevanti ed emergenti in contrasto con l'universalismo e cosmopolitismo settecentesco o più propriamente illuministico» ( $\mathrm{p}$. 9).

2 Il libro è strutturato intorno a quattro nuclei tematici fondamentali: nella trilogia di studî che forma la prima sezione dell'opera («Le Città dell'infanzia»), l'autore focalizza la sua attenzione sulla dialettica tra infanzia e memoria concentrandosi, in un primo momento, sulla funzione della minuzia mnemonica di Rousseau nella sua celebrazione 
della poesia dell'infanzia espressa attraverso la descrizione di Ginevra nel primo capitolo delle Confessions. Se è vero, scrive Prosio, che Ginevra «è stata la prima città della letteratura mondiale che scrittore abbia mai investito della poesia della propria infanzia», è altrettanto significativo il fatto che Rousseau impedisca al lettore di «vederla del tutto come un dorato e vagheggiato ricetto» (p. 26). Ai temi della nascita, dell'infanzia e della morte è visualmente e spiritualmente legata la rappresentazione di Saint-Malo nei Mémoires d'Outre-Tombe di Chateaubriand. In particolare, è la tomba dello scrittore al Grand-Bé a racchiudere la più viva forza evocativa rispetto agli altri spazi chateaubriandiani di Saint-Malo: i luoghi d'infanzia e la dimora di morte evocate dallo scrittore fanno, di Saint-Malo, «il simbolo di una vita romantica [...] di cui egli volle sottolineare la portata mitica ed insistere sulla sua unicità» (p. 34). Diversamente da Rousseau e da Chateaubriand, Stendhal mostra, nella Vie de Henry Brulard, una insensibilità fredda e quasi cinica nei confronti di Grenoble (città che lo scrittore evoca come «le souvenir d'une abominable indigestion»): uomo e scrittore del presente, votato all'egocentrismo, che soltanto in Milano riconosce il luogo e il tempo della felicità, Stendhal, osserva Prosio, «manca assolutamente di prospettiva storica» (p. 48) e psicologica. La totale mancanza d'amore per la sua città d'infanzia si risolve, quindi, «in una scarsa visibilità letteraria della stessa nella sua pagina» (pp. 52-53).

3 «Tra laghi e montagne» è il tiolo della sezione che raccoglie tre capitoli dedicati rispettivamente a Rousseau, a Lamartine e a Shelley. Luoghi di cesura netta col mondo, il Lago di Bienne e l'Isola di Saint-Pierre evocati nella quinta Promenade delle Rêveries $d u$ promeneur solitaire sono messi a confronto con la seducente (e specularmente opposta) rappresentazione delle Alpi nella Nouvelle Héloise. Sulla nuova immagine delle Alpi nel primo Romanticismo francese vista in relazione con il mutamento del sentimento della natura nella sua rappresentazione letteraria, è dedicato il capitolo centrato allo studio del poema Jocelyn di Lamartine (con riferimenti anche al Guglielmo Tell di Rossini). Luogo di separazione dalle brutture e dalle cattiverie del mondo, la montagna riveste, in Lamartine, «una funzione catartica» (p. 73): la durezza e le privazioni esterne si fanno motivo di pace, di letizia e di una conquistata serenità. Il legame tra Monte Bianco e romantici inglesi è analizzato nella parte dedicata a Mary Shelley e alla poesia romantica inglese che chiude questa seconda trilogia di saggi.

Gli studî che formano la sezione centrale dell'opera ("Cinque romanzi, cinque paesi») riguardano due scrittori di lingua francese (Barbey d'Aurevilly e Rodenbach), due romanzieri inglesi (E. Brontë e Stevenson) e lo scrittore italiano Edoardo Calandra. Nell' Ensorcelée, «ce livre [...] profondément normand» che Barbey d'Aurevilly pubblicò nel 1854 , lo scenario geografico rappresenta il vero elemento unificante dei vari momenti che compongono la vicenda narrata: è la prima volta che, nell'ottocento, «la Normandia fa la sua autorevole comparsa in un'opera narrativa» (p. 105) nella quale l'elemento fantastico prende il sopravvento, dal punto di vista topografico, sentimentale e mentale, sul programma di ricostruzione storica. In Bruges-la-morte (1892) dello scrittore belga Georges Rodenbach, è la città come "état d'âme» a essere assunta come personaggio centrale del testo, come sostanza e giustificazione del romanzo. Anche in Wuthering Heights, la brughiera e lo Yorkshire descritti da Emily Brontë incarnano uno stato d'animo e il paesaggio descritto assume una funzione unificante nella struttura del romanzo. Weir of Hermiston di Stevenson rappresenta un vero e proprio inno di nostalgia dello scrittore (che compose l'opera nelle Isole Samoa) per la Scozia; ne La Bufera di E. Calandra emergono quei tratti di conservatorismo sentimentale e psicologico per la dignità di un certo mondo pre-rivoluzionario (il caro 
«Vecchio Piemonte»), certamente antiquato, forse superato, ma sempre degno di rispetto se non di approvazione.

5 «Tre Capitali: Roma, Parigi, Londra» e quattro scrittori: Hawthorne (The Marble Fun), Flaubert (L'Éducation sentimentale), Stevenson (The strange case of Dr. Jekyll and Mr. Hyde) e Dickens (A Christmas Carol) sono esaminati da Prosio nella parte finale dell'opera. In questi studî, l'interesse dell'autore si focalizza sulle modalità attraverso cui la trama e le atmosfere umane e ambientali presenti nelle opere esaminate si inseriscono nelle differenti cornici urbane entro cui si svolgono e si esplicitano le dinamiche dei testi. Particolarmente interessanti sono, a nostro giudizio, i rilievi dell'autore, a proposito del rapporto narrativo tra Parigi e Madame Arnoux e il confronto tra la rappresentazione della capitale francese in Flaubert e in Balzac. La Parigi che fa da sfondo all'Éducation sentimentale si muove in tutt'altra aura dalla Parigi di Balzac: «lo sguardo allegro e distaccato, sicuro e onnicomprensivo di Balzac» è sostituito, nel romanzo di Flaubert, «da un avvicinarsi trepido e circoscritto, assolutamente soggettivo» (p. 179). 